\title{
Student attitudes regarding the educational value and welfare implications in the use of model eyes and live dogs in teaching practical fundus examination: evaluation of responses from 40 students
}

\author{
D.L. Williams ${ }^{1, *}$, C. Wager ${ }^{2}$ and J. Brearley ${ }^{2}$ \\ ${ }^{I}$ Queen's Veterinary School Hospital, Department of Veterinary Medicine, University of Cambridge, Madingley \\ Road, Cambridge CB3 OES, UK \\ ${ }^{2}$ Clinical Skills Center, Department of Veterinary Medicine, University of Cambridge, Madingley Road, Cambridge \\ CB3 OES, UK
}

\begin{abstract}
This study sought to document student opinions on the educational value and welfare implications of use of artificial model eyes and live dogs in the training of veterinary students in examination of the canine fundus. Forty students who had undertaken a practical class on canine fundoscopy involving both use of artificial model eyes and live dogs were asked to complete a short questionnaire using a Likert scale to gauge their opinion on whether the use of live dogs and artificial eyes was very valuable (scoring 2), valuable (1), a neutral response (0), not particularly valuable (-1) or not at all valuable (-2) and to write a free text response on their views of the educational value and welfare implications of using artificial model eyes or live dogs in training for ophthalmic examination of the canine ocular fundus. Likert responses were $1.84 \pm 0.37$ for using live greyhounds and $0.58 \pm 0.79$ for using simulator eyes $(\mathrm{p}<0.0001)$. Thematic analysis of the written responses showed that while the artificial eyes were considered somewhat valuable in initial training, the live dogs were significantly preferred for their realism and the opportunity to examine the eye while handling a live animal. In conclusion, while model eyes are valuable initial training in use of the ophthalmoscope for funduscopic examination, students consider that examining the eye in the live dog is significantly more valuable and that the welfare of dogs thus used is not in their view unduly compromised.
\end{abstract}

Keywords: Education, Fundoscopy, Model eye, Ophthalmoscopy, Student.

\section{Introduction}

Examination of the ocular fundus is initially a difficult task especially in clinically affected animals. Students generally report it to be invaluable to practice ophthalmoscopy on live normal dogs before joining a clinic to evaluate animals with diseased eyes. Yet concerns may be raised over the use of live dogs to train veterinary students in ophthalmic examination since it might be argued that animals may be compromised by having inexperienced undergraduates using ophthalmic equipment with which they are unfamiliar. Here we sought to ask students for their opinions on the educational value and potential ethical issues regarding use of live dogs versus model eyes in training them to examine the ocular fundus. A significant conundrum in much undergraduate veterinary education involves the fact that many techniques which are essential to master require the involvement of live animals in student teaching. Such involvement can adversely affect the welfare of the animals, especially where many students need to examine a relatively small number of animals. This gives potential stress to the animals and, it has to be said, to the students also for whom the care of the animals being used is paramount. But as Aristotle said, the best way to learn how to build a wall, or to play the lyre, is actually to build the wall or play the lyre (Aristotle, $350 \mathrm{BCE}$ ). A theoretical approach to learning a practical task often teaches little - what is needed is practice at the task itself. Models can be developed to aid in the first stages of learning a new technique or skill. The development of the clinical skills lab has been highly valuable in doing just this, with many valuable resources provided from vascular models for practising venepuncture (Eichel et al., 2013), through haptic cows to master rectal examination skills (Baillie et al., 2005) to the virtual stethoscope to educate in cardiac auscultation (Fuentes et al., 2015). Recently a model eye has been developed and validated to aid in the development of skills in ophthalmic examination (Nibblett et al., 2015). The model was constructed using a table tennis ball and a 20D hard contact lens with the inside of the ball painted black with a triangle of coloured paper to model the tapetal fundus and a white circle to represent the optic disc. The eye was mounted on a hardwood silhouette of a dog's head. Six veterinary ophthalmologists and a further 16 members of the educator faculty were asked to rank a number of statements regarding the appropriateness, realism and ease of use from strongly agree to strongly disagree. The participants 'overwhelmingly either 
agreed or strongly agreed' regarding the construction, ease of use of the model, suitability for teaching, helpfulness before fundoscopy in the live animal and effectiveness for assessment of skill in fundoscopy, but fewer agreed or strongly agreed regarding the realism of the model. The performance of 29 students was assessed, these individuals being divided into a group which used the model eye practising direct and indirect ophthalmoscopy on the eye for 10 minutes and a group which did not. Each group then received a 1.5 hour practical teaching session involving live dogs. At the end of this the students' ability at fundoscopy was evaluated by using a modified model eye with a coloured shape affixed at the point of the optic nerve. The ability of students to recognise the shape and the time taken were compared between the group that had used the model eye and those that had not. In that study, however, the students were not asked for their opinion on the educational value of the model eyes or the live dogs in practising fundoscopy and thus here we aimed to ask students who had used both model eyes and live dogs in funduscopic training for their opinions on both training aids.

A model system such as this clearly has benefits in reducing the use of live animals for the initial stages of learning ophthalmic examination. Yet such a model eye is relatively far removed from a real eye in a live dog with regard to its anatomical and pathological appearance and the training that is needed to examine the eye in a live animal. Here we sought to ask students their opinion on the use of the model eye and the live dog for learning ophthalmic examination skills. We asked students to grade their opinions both using a Likert scale response and a free text response. Evaluation of these student responses involved quantitative analysis of the Likert scale answers and a more qualitative thematic assessment of the free text responses. This mixed methods methodology allows a detailed and yet at the same time deep analysis of the student opinions with regard both to the educational value and the welfare implications of using a model eye in the clinical skills centre and live animals in the veterinary clinic.

\section{Materials and Methods}

The study was approved by the ethics and welfare committee of the department and the dogs were at all times kept in full compliance with the department's welfare criteria which comply fully with national guidelines on the care and use of laboratory animals. All students questioned in the study gave full informed consent to their opinions being evaluated in this study and their involvement was approved by the departmental welfare and ethics committee.

The first 40 students undertaking the ophthalmoscopy practical class were asked for their opinions on the educational value and ethical appropriateness of using live blood donor greyhounds and model simulator eyes in training to perform ophthalmoscopy on clinical patients. As a practical part of their fourth year ophthalmology training, students receive a morning session in groups of 4-5 students first using two blood donor greyhounds and also model eyes in the clinical skills laboratory (Fig. 1) to practise direct and indirect ophthalmoscopy using a direct ophthalmoscope (Keeler Practitioner, Windsor UK) for one hour before joining an ophthalmology clinic for the rest of the morning where they examine clinical cases.

Students are shown how to use the direct ophthalmoscope at 0 dioptres for distant direct ophthalmoscopy and then close fundoscopy, then at 10 dioptres to evaluate the lens and iris surface and at 20 dioptres to evaluate the adnexa and cornea. Students are instructed to adjust the rheostat to a low light level and each student examines the eye for up to but not exceeding one minute at a time. The greyhounds used are well used to being handled by the students and this do not need to be sedated or restrained for the class. Emphasis is placed on acquisition of experience in direct ophthalmoscopy as every veterinary clinic will have a direct ophthalmoscope but not necessarily equipment for binocular indirect ophthalmoscopy. Each students is also shown how to use an indirect loupe lens (Volk, Tokyo Japan) with a pentorch light source to demonstrate monocular indirect ophthalmoscopy and also the chance to use a binocular indirect ophthalmoscope (Keeler Vantage, Windsor UK) to show the difference in size of visual field with this technique. This means that each dog is examined by four students during the practical session which lasts for around one hour. The model eyes were purchased from a supplier (ProMedica UK Ltd, Bristol UK) and were placed in the head of a model dog (Fig. 1). While not particularly realistic from the perspective of the fundus examination, they have a replica optic nerve and coloured lines similar to retinal vessels (Fig. 2) with words at the end of the vessel (Fig. 3), allowing assessment of whether the student is truly observing features in the posterior segment by asking what word

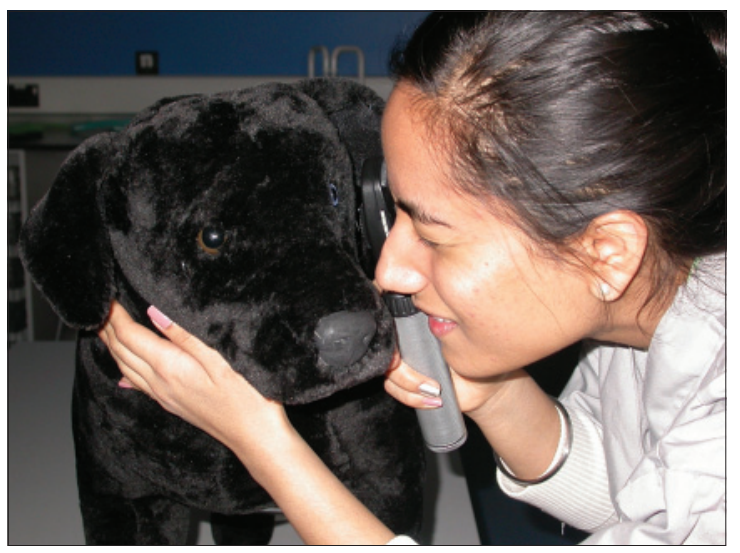

Fig. 1. Student using model eye to practise fundoscopy. 


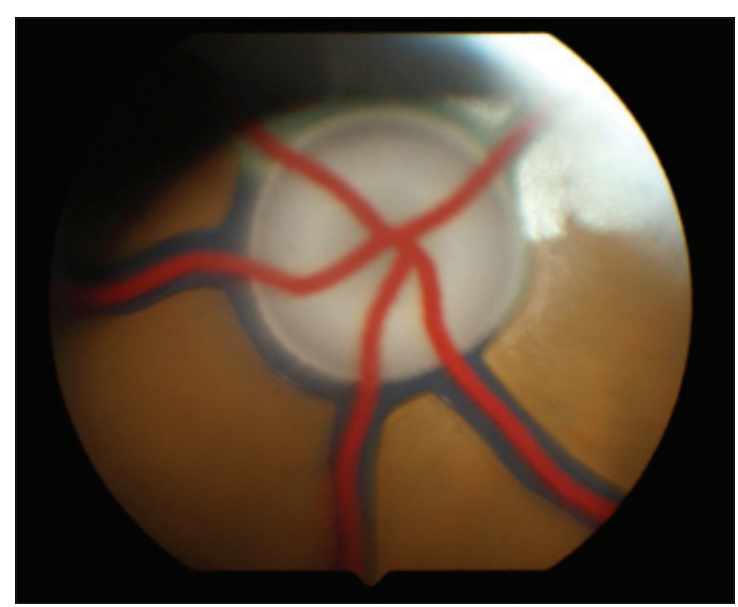

Fig. 2. Optic nerve and blood vessels in the model fundus.

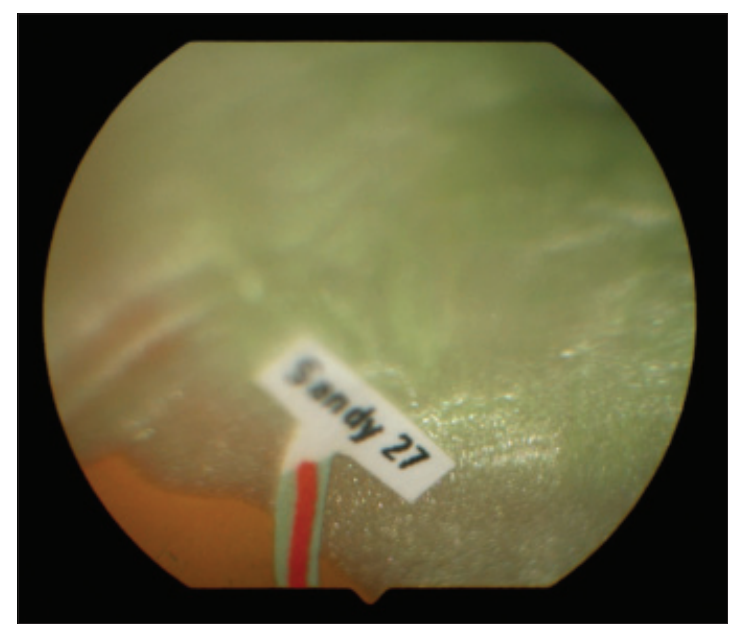

Fig. 3. Text at end of blood vessel in model eye.

they can visualise in the fundus. Following use of these model eyes, the students are given the opportunity to examine the eyes of blood donor greyhounds housed in the veterinary school for half an hour before examining animals being evaluated in a set of clinical consultations in the hospital.

At the end of the full session including examination of normal greyhounds and evaluation of clinical cases students were asked to reflect on their experiences through the morning. They were first asked to score their opinions on the educational value of the use of the model eyes and live dogs on a Likert scale from very valuable $(+2)$, valuable $(+1)$ not particularly valuable $(-1)$ or not at all valuable $(-2)$ with 0 as a neutral response. Next they were given the opportunity to write a free text response on the educational value and ethical justification for both techniques. The Likert scale responses were summated and are presented as mean \pm standard deviation while the free text responses were subject to a qualitative thematic analysis. Differences between the Likert responses for model eyes and live dogs were compared with a paired Students $t$ test with significant difference deemed to have been reached at $p<0.05$. The text responses were thematically coded by the authors by reading and re-reading the responses, searching for themes showing different reasons for valuing or otherwise the use of model eyes or live dogs as detailed in standard educational research texts (Guest et al., 2012).

Regarding the size of the sample of student chosen, it was not possible to define an a priori sample size as the range of views were not known before the study began, and thus the size of sample required was determined by assessing when a thematic saturation was achieved (Guest et al., 2006). The responses were gathered over 10 weeks as each student group involved four individuals. The responses gained from the last 10 students did not significantly change the mean Likert scale values gained from the previous eight weeks of classes. At this stage of the student evaluation the thematic analysis had also reached saturation with no new ideas or opinions expressed and thus the study was considered completed.

\section{Results}

The Likert scale responses were $1.84 \pm 0.37$ for using live greyhounds and $0.58 \pm 0.79$ for using simulator model eyes, these values differing at $p<0.0001$. The thematic analysis of the text responses showed a number of recurring responses as shown in Table 1 . The predominant comments indicated that while the artificial eyes were beneficial to practice using the ophthalmoscope, the live dogs were substantially better for several reasons. First the eye of the live greyhound was, not surprisingly, more realistic that the model eye (19 responses). Students considered it valuable to identify structures in the real eye of a live animal (19 responses). Six students found it easier to identify structures in the living animal while 15 did not find it easy. Artificial eyes allowed the students more time to practise the relevant technique ( 8 responses) and did not move making the initial use of the ophthalmoscope easier ( 7 responses). The very fact that the animals were moving and required restraint while examining the fundus was on the one hand seen by many students as a beneficial factor in training in ophthalmic examination, but on the other a potentially detrimental feature. As one student wrote: "it was good to practice [sic] how to hold head while examining the dog although it may be more difficult to see when dog is moving'. In the words of another: 'The dogs are alive so move just like real patients which is useful for getting orientation within the eye but not always the easiest to get an image in the eye'. A third respondent had a similar mixed perspective: 'The eye is different in the live dog so it was good to see this. In time I managed to see everything. Also gives the opportunity to practice examining when the dog moves 
Table 1. Thematic analysis of material from free text responses from 40 students regarding student opinions on use of live greyhounds and model eyes for training in fundoscopy.

\begin{tabular}{lclc}
\hline Live greyhounds & $\begin{array}{c}\text { Number of } \\
\text { responses }\end{array}$ & Model eyes & $\begin{array}{c}\text { Number of } \\
\text { responses }\end{array}$ \\
\hline More realistic than model eye & 19 & Not realistic & 9 \\
Good to examine a real eye & 19 & Benefit of immobile eye & 7 \\
Easier to identify ocular structures in live animals & 6 & $\begin{array}{l}\text { Can be used at any time } \\
\text { Can take as much time as one needs to } \\
\text { examine eye }\end{array}$ & 8 \\
Not easy to identify ocular structures in live animals & 15 & Words at back of eye help & 3 \\
Stress may be caused to animals & 10 & No normal anatomy & 2 \\
Dogs enjoyed attention & 4 & & \\
\hline
\end{tabular}

though it's not always easy to see everything in the eye when the dog won't keep still'.

Ten students $(25 \%)$ commented on possible welfare issues involved in using live animals with comments such as 'The eyes in the real dog are realistic and they might have abnormalities to see but you feel bad you have to keep messing them around and can't manipulate them as easily as an artificial eye' and 'Really useful for seeing all structures but dogs get fed up after a few minutes'. Others noted that the dogs were not, in their opinion, stressed while others looked beyond this short period of handling with comments such as 'even though these dogs don't get stressed others might, so with other things through the day this could be cumulatively stressful'. One student reflected: 'These are easy dogs to examine and good to get the chance to look without any owners present but are the greyhounds happy?'. These animals are ex-racing dogs which are used both as blood donors and for numerous practical classes from clinical anatomy classes positioning for radiography so the issue concerning their overall welfare is a valid one but well covered through our ethics and welfare provision through the veterinary school. As one of the students observed: 'the greyhounds get an outing too so there are no disadvantages!'.

\section{Discussion}

Concern over the detriment to animal welfare that may result from use of animals in the training of veterinary students has been a significant issue in veterinary education for many years. Yet the benefits from students interacting with live animals as they learn diagnostic and therapeutic strategies cannot be denied. In defining the balance between these two potentially contradictory elements in veterinary education, it is interesting and potentially worrying to note that few studies have asked the students themselves what their attitudes are. Here, in a relatively small and preliminary manner, we sought to hear the student voice with regard to the costs and benefits of using live dogs or model eyes in the training of students to examine the fundus of the globe using an ophthalmoscope.
The Likert scale responses showed that students considered use of live greyhounds significantly more educationally valuable than model eyes. These differed from the Likert scale responses from ophthalmologists and educators in Nibblett and colleagues' study (Nibblett et al., 2015) validating a model for teaching fundoscopy. Those respondents considered the model eye appropriate, suitable for teaching and evaluating students and helpful to those students prior to examining live animals, rather different opinions from the students in the present study. It has to be said that these ophthalmologists and educators in Nibblett's study were not given the opportunity to compare the model eye and live dog, neither were the students in that study given the chance to voice their opinion.

The overall student opinion in the present study was that while the model eyes were valuable for practising initial use of the ophthalmoscope, eyes of live dogs were considerably more realistic than the model eyes and that the experience of examining the eyes of a live animal which required careful restraint was substantially more valuable than using the model eyes. This is not to say that model eyes are worthless by any many of means. It is just that, as for the entire clinical skills center, the model eyes are not a replacement for using living animals, but rather a valuable first step in the process of learning fundoscopy. Students recognised that the model eyes allowed them greater time to practise and were available at any time, but using the live dogs was essential fully to become competent in examination of the canine retina. It was only with live dogs that the technique of fundoscopy was properly embedded in the wider context of dealing with the whole animal. Only the examination of live dogs allows integrating the clinical skill of using the ophthalmoscope with what we might call the art of calming a nervous dog. Only being integrated into the ophthalmic clinic allows students to talk to the owner as one examines the eye, linking the practicalities of what one is seeing in the retina with the science one was taught in the classroom and through reading the 
textbook. This takes us back to the very definition of a clinical skill. Many would say it is much more than a mere technical ability (Michels et al., 2012). Rather, it involves a cognitive approach to understanding as the foundation for context-specific practical actions, as outlined by Hubert Dreyfus, (Dreyfus, 2006; Carraccio et al., 2008) among others. The model eye allows a basic acquisition of the fundamentals of using the direct ophthalmoscope, but the skill of using it in a clinical setting requires much more than that. Indeed, for many years veterinary ophthalmology was taught using live dogs alone and without first using model eyes. So when the model eye was introduced we thought it is important to ask the students for their opinions on this new approach.

Assessing students' attitudes to methods used in their education is today considered central to improving the pedagogy that is the foundation of their learning. Many in tertiary education now see students primarily as consumers (Hill, 1995) with a commensurate requirement to define what their needs and aspirations are and how this should shape the learning experience. And yet for many years the student voice was simply unheard, indeed never listened to. The teacher surely was the one who knew how to teach; the students were there merely as recipients of the lecturer's wisdom. Today however, seeking to access students' opinions is seen as an integral part of the development of pedagogy. The medical fraternity have for many years evaluated student views on the benefits of virtual or model systems in their education (Bearman, 2003; Martens et al., 2009; Lund et al., 2011) while similar assessment of the veterinary student voice with regard to using such systems has been less frequently reported (Howe et al., 2005; Langebæk et al., 2016). Specifically concerning model eyes in the teaching of ophthalmoscopy, the TOTeMS (teaching ophthalmoscopy to medical students) study showed that $71 \%$ preferred learning ophthalmoscopy using humans than model eyes while $77 \%$ preferred colour fundus photographs for learning pathological features of the human fundus (Kelly et al., 2013). Here we sought to evaluate what educational value for students the model eyes had compared to using live dogs, and sought to gauge student views on the welfare implications of using the live dogs in this practical teaching class and the benefits of using model eyes in developing this clinical skill. Their opinions were that the model eyes were valuable as an initial phase in teaching ophthalmoscopy, but could in no sense replace the use of live dogs prior to student involvement in the ophthalmology clinic.

A final issue concerns which ophthalmoscopic techniques should be taught to veterinary students. Referral ophthalmologists generally use indirect ophthalmoscopy and slit lamp biomicroscopy today, and yet the equipment for these techniques is not generally available in first opinion veterinary clinics. Thus direct ophthalmosopy is the standard technique used in first opinion clinics and therefore the fundscopic method on which the ophthalmic education of undergraduates concentrates, at least in Cambridge veterinary school. The same is true of ophthalmic education of medical undergraduates, although there is some debate as to whether direct ophthalmoscopy should be part of their training. The question 'should direct ophthalmoscopy be taught to undergraduate medical students' led to responses both positive (Yusuf et al., 2015) and negative (Purbrick and Chong, 2015). But Purbrick and Chong (2015) answering 'no' suggested not that more complex instruments such as indirect ophthalmoscopes or slit lamp biomicroscopes be used but that students should be given fundus photographs to show the retinal lesions involved rather than demonstrating the techniques themselves. Yusuf et al. (2015) answering 'yes' stated that 'direct ophthalmoscopy adds a valuable weapon in the diagnostic armamentarium of the clinician.' If that is the case in human medicine where referral to a specialist is rapid and easy, it is all the more important in veterinary medicine where fewer owners have the financial option or geographical proximity to be able to be referred with ease - it is vital that new graduates have a basic grasp of ophthalmic examination.

Of course such a first chance to use the direct ophthalmoscope on a model eye and a live dog is merely the beginning. One would hope that this first experience enthrals the students, as it enthralled the senior author of this publication thirty years ago, leading to a lifelong passion for ophthalmology. It is merely the first step to many opportunities to examine the beautiful canine fundus. Also, perhaps this is the most exciting difference between the model eye and the live animal. One often hears exclamations 'wow!' 'amazing!' 'beautiful!' when students first see the canine fundus. It has to be said that these exclamations are not heard when students view the model eye. Education should provide more, much more, than a transfer of knowledge and skill; it should stimulate interest and enthusiasm. And a first view of the canine fundus can readily provide just such an opportunity.

\section{References}

Aristotle. 350 BCE. Nicomachean Ethics, Book II.

Baillie, S., Crossan, A., Brewster, S., Mellor, D. and Reid, S. 2005. Validation of a bovine rectal palpation simulator for training veterinary students. Stud. Health Technol. Inform. 111, 33-36.

Bearman, M. 2003. Is virtual the same as real? Medical students' experiences of a virtual patient. Acad. Med. 78, 538-545.

Carraccio, C.L., Benson, B.J., Nixon, L.J. and Derstine, P.L. 2008. From the educational bench to the clinical bedside: translating the Dreyfus 
developmental model to the learning of clinical skills. Acad. Med. 83, 761-767

Dreyfus, H. 2006. From novice to world discloser. Paper presented at the Accreditation Council for Graduate Medical Education Design Conference on the Learning Environment; September 9, 2006; Chicago, Ill.

Eichel, J.C., Korb, W., Schlenker, A., Bausch, G., Brehm, W. and Delling, U. 2013. Evaluation of a training model to teach veterinary students a technique for injecting the jugular vein in horses. J. Vet. Med. Educ. 40, 288-295

Fuentes, V.L., Trace, C., Powney, S., Boswood, A. and Cox, B. 2015. Development of a Veterinary Virtual Stethoscope. Available at: http://www.live.ac.uk/ Media/LIVE/PDFs/LIVE\%20TEACHING\%20 DEVELOPMENT\%20TEAM\%20PRIZE\%20 2013\%20Virtual\%20stethoscope\%20vlf\%20(2).pdf

Guest, G., Bunce, A., and Johnson, L. 2006. How many interviews are enough?: An experiment with data saturation and variability. Field Methods 18, 59-82

Guest, G., MacQueen, K.M. and Namey, E.E. 2012. Applied thematic analysis. Thousand Oaks, California: Sage, pp: 11.

Hill, F.M. 1995. Managing service quality in higher education: the role of the student as primary consumer. Qual. Assur. Educ. 3, 10-21.

Howe, L.M., Boothe, H.W. Jr. and Hartsfield, S.M. 2005. Student assessment of the educational benefits of using a CD-ROM for instruction of basic surgical skills. J. Vet. Med. Educ. 32, 138-143.
Kelly, L.P., Garza, P.S., Bruce, B.B., Graubart, E.B., Newman, N.J. and Biousse, V. 2013 Teaching ophthalmoscopy to medical students (the TOTeMS study). Am. J. Ophthalmol. 156, 1056-1061.

Langebæk, R., Tanggaard, L. and Berendt, M. 2016. Veterinary Students' Recollection Methods for Surgical Procedures: A Qualitative Study. J. Vet. Med. Educ. 43(1), DOI: http://dx.doi.org/10.3138/ jvme.0315-039R1.

Lund, B., Fors, U., Sejersen, R., Sallnäs, E.L. and Rosén, A. 2011. Student perception of two different simulation techniques in oral and maxillofacial surgery undergraduate training. BMC Med. Edu. 11, 82. doi: 10.1186/1472-6920-11-82.

Martens, M.J., Duvivier, R.J., van Dalen, J., Verwijnen, G.M., Scherpbier, A.J. and van der Vleuten, C.P. 2009. Student views on the effective teaching of physical examination skills: a qualitative study. Med. Educ. 43, 184-191.

Michels, M.E., Evans, D.E. and Blok, G.A. 2012. What is a clinical skill? Searching for order in chaos through a modified Delphi process. Med. Teach. 34, e573-581.

Nibblett, B.M., Pereira, M.M., Williamson, J.A. and Sithole, F. 2015. Validation of a Model for Teaching Canine Fundoscopy. J. Vet. Med. Educ. 42, 133-139.

Purbrick, R.M. and Chong, N.V. 2015. Direct ophthalmoscopy should be taught to undergraduate medical students--No. Eye (Lond). 29, 990-991.

Yusuf, I.H., Salmon, J.F. and Patel, C.K. 2015. Direct ophthalmoscopy should be taught to undergraduate medical students-yes. Eye (Lond). 29, 987-989. 